\title{
Heat Generation by Polypyrrole Coated Glass Fabric
}

\author{
A. M. Rehan Abbasi, ${ }^{1,2}$ J. Militky, ${ }^{1}$ and J. Gregr' \\ ${ }^{1}$ Technical University of Liberec, Studentska 2, 46117 Liberec, Czech Republic \\ ${ }^{2}$ Department of Textile Engineering, BUITEMS, 87100 Quetta, Pakistan \\ Correspondence should be addressed to A. M. Rehan Abbasi; rehan_abbaci@hotmail.com
}

Received 6 January 2013; Revised 19 June 2013; Accepted 2 August 2013

Academic Editor: Shuichi Tanoue

Copyright (C) 2013 A. M. Rehan Abbasi et al. This is an open access article distributed under the Creative Commons Attribution License, which permits unrestricted use, distribution, and reproduction in any medium, provided the original work is properly cited.

\begin{abstract}
Vapor deposition technique was employed to coat polypyrrole (PPy) on glass substrate using $\mathrm{FeCl}_{3}$ as oxidant and p-toluenesulfonic acid ( $\left.{ }^{-} \mathrm{OTs}\right)$ as doping agent. The Joule heating effect of PPy coated E-glass fabric was studied by supplying various DC electric fields. The coated fabric exhibited reasonable electrical stability, possessed medium electrical conductivity and was effective in heat generation. An increase in temperature of conductive fabric subjected to constant voltage was observed whereas decrease in power consumption was recorded. Thickness of PPy coating on glass fibers was analyzed by Laser confocal microscope and scanning electron microscope.
\end{abstract}

\section{Introduction}

In the late 1970s, MacDiarmid, Heeger, and Shirakawa discovered how to get polymers conducting electricity [1]. The first material becoming an intrinsically conducting polymer (ICP) was polyacetylene, after a doping with iodine. The announcement of this discovery quickly reverberated around scientific communities, and intensity of the research for other conducting polymers magnified dramatically [1-3]. A new generation of polymers was then developed, exhibiting the electrical and optical properties of metals or semiconductors, at the same time retaining the attractive mechanical properties and processing advantages of polymers. Intrinsically, conducting polymers were immediately seen as a new route to mimic metallic conductivity, besides the well-known approach to insert conductive fillers into an inherently insulating resin, or to coat a plastic substrate with a conductive metal solution [4].

Ability to apply conducting polymers to any fiber type and textile structure provides unlimited flexibility in the design of conductive fabrics. Each fiber on the surface of the textile is coated by a thin layer of conducting polymer. As a result of chemical polymerization in the presence of a fabric, a continuous thin film of conducting polymer is formed on the fabric surface. Current will flow through these fabrics without any wiring through them and the heat generated is not affected by holes or cuts in the fabric as the entire surface is electrically conductive.

Wide ranging modulation of electrical properties of conducting textiles leads to various other applications. Electrical conductivity is sensitive to external stimuli such as temperature and pressure, suggesting applications in the area of sensors.

Kinetics of degradation of conductivity of chemically and electrochemically synthesized polypyrrole at elevated temperatures has been thoroughly investigated. However, only a few brief reports have been published on the aspect of heat generation. In one of these reports, low temperature radiant heating panels were produced by PPy coating, and effect of heat generation was studied, but their applications are still limited due to comparatively low tensile strength of the fabric. The aim of this work is to develop heating panels by coating PPy on glass fabric for commercial and industrial applications, specially suggested for space heating systems at low cost. The reason for choosing glass fiber is its higher tensile strength compared to other fibers of the same price.

\section{Methodology}

2.1. Sample Preparation. PPy was coated on E-glass fabric having specifications given in Table 1 . The glass fabric was first immersed in aqueous solution of ${ }^{-} \mathrm{OTs}$ and $\mathrm{FeCl}_{3}$ as 
TABLE 1: Specification of the E-glass fabric used for experiment.

\begin{tabular}{lc}
\hline Properties & Index \\
\hline Weave & Plain \\
Fineness (tex) & \\
$\quad$ Warp & 1250 \\
Weft & 1250 \\
Warp/cm & 3.67 \\
Weft/cm & 3.5 \\
Cover factor (SI) & \\
$\quad$ Warp & 13 \\
$\quad$ Weft & 12.4 \\
Areal density $\left(\mathrm{g} \cdot \mathrm{m}^{-2}\right)$ & 900 \\
Thickness $(\mathrm{mm})$ & 1.1 \\
Fabric porosity $(\phi)$ & 0.678 \\
\hline
\end{tabular}

an oxidant in molar ratio $1: 2$, respectively, at the temperature of $10^{\circ} \mathrm{C}$ and squeezed with rubber rollers padding machine with $100 \%$ pickup.

Fabric porosity can be calculated by

$$
\phi=1-\left(\frac{\rho_{\mathrm{fa}}}{\rho_{\mathrm{f}}}\right) .
$$

Here, $\rho_{\mathrm{fa}}$ is the density of fabric that can be evaluated through dividing areal density by the thickness, whereas $\rho_{\mathrm{f}}$ is the known density of fiber.

As the amount of PPy depends upon the oxidizing agent present in the system, different specimens were developed by taking different concentrations of $\mathrm{FeCl}_{3}$ as $0.3,0.4,0.5$, and $0.6 \mathrm{~mol} / \mathrm{L}$ keeping dopant to oxidizing agent ratio as $1: 2$ constant.

If released to air, a vapor pressure of $8.35 \mathrm{mmHg}$ at $25^{\circ} \mathrm{C}$ indicates pyrrole exists solely as a vapor in the ambient atmosphere. Therefore, fabric was then immediately exposed to vapors of pyrrole monomer for 8 hours. Later, fabric was rinsed with ethanol and distilled water to remove excess of monomer and biproducts. Finally, it was dried in air at $25 \pm 4^{\circ} \mathrm{C}$ for 24 hours. The formation of the conductive polymer coating on the textile substrate starts from deposited oligomers, which form islands in the initial stage and finally grow and cover the surface of the substrate completely [5].

For evaluation of electrical properties of fabrics, two parameters were selected, namely, the surface and volume resistivity. These parameters can be calculated from the information of surface and bulk resistivity, the dimensions of the electrodes, and/or the thickness of the sample. For this purpose, concentric electrode system was used conforming to DIN EN 1149-1, EN 100 015, EN 61340-5-1, together with Agilent 53131A digital multimeter. Measurements of electrical resistance were performed in the climatic conditions $t=$ $20 \pm 2^{\circ} \mathrm{C}$ and $\varphi=40 \pm 2 \%$, which is in accordance with the standard ČSN 800059.

The morphology and thickness of PPy coating on glass fiber was characterized by scanning electron microscope (SEM) Carl Zeiss Ultra plus after gold coating and Laser confocal microscope in the Technical University of Liberec, Czech Republic.
2.2. Temperature Measurements. For the purpose of evaluating heat generation by polypyrrole coated glass fabric samples, $5 \mathrm{~V}, 10 \mathrm{~V}, 15 \mathrm{~V}$, and $20 \mathrm{~V}$ DC electromotive forces by DC voltage supplier NZ-2229.2 from Statron, Czech Republic, were applied via stainless steel clamp type electrodes. Meanwhile DC current passing through the substrate was measured by connecting Agilent 53131A digital multimeter in series. The rise in temperature was determined by the infrared thermal camera by Fluke together with k-type thermocouples in connection with ART data acquisition module. The infrared thermal camera was helpful to figure the homogeneity of the specimen out.

\section{Results}

Oxidation of the pyrrole monomer in the presence of the substrate causes polymerization to take place on the fabric surface, resulting in a thin and smooth conductive coating, whilst some of the polymer particles formed on the PPy coating deposited on the surface at prolonged reaction time and at higher monomer concentration, which can be seen as clumps of PPy deposition on the surface and shown as inhomogeneity in the PPy coating, as shown in Figure 1. The morphology of the glass surface after coating with PPy shown in Figure 2 explains that each fiber in the fabric structure is covered with PPy depending upon the concentration. The average thickness of coating of PPy on E-glass fiber was measured as $1.165 \pm 0.05 \mu \mathrm{m}$.

The average thickness was also measured with the help of Laser confocal microscopy and was found to be around $1.372 \mu \mathrm{m}$ as shown in Figure 2, which also proves the homogeneity and smoothness of PPy coating on glass fibers.

The weight gain $(\Delta m)$ by the glass fabric after coating follows a linear function of concentration of $\mathrm{FeCl}_{3}(C)$ as revealed in Figure 3, and this relation can be expressed mathematically as (2) with coefficient of determination $R^{2}=$ 0.962 :

$$
\Delta m=9.0442 \cdot C .
$$

The presence of PPy on the E-glass fabric specimen was characterized by Fourier transform infrared spectroscopy (FTIR) as shown in Figure 4. The strong absorbance of control specimen at $958 \mathrm{~cm}^{-1}$ shows the occurrence of $\mathrm{Si}-$ $\mathrm{O}-\mathrm{Si}$ and $\mathrm{Si}-\mathrm{OH}$ in E-glass structure. The absorption at 1546 and $1452 \mathrm{~cm}^{-1}$ assigned to ring vibration of $\mathrm{C}=\mathrm{C}$ and $\mathrm{C}=\mathrm{N}$ as well as at $1168 \mathrm{~cm}^{-1}$ for $\mathrm{N}-\mathrm{C}$ vibration confirms the presence of pyrrole ring of PPy. Absorptions at 1303 and $1035 \mathrm{~cm}^{-1}$ confirm in-plane vibration of $=\mathrm{C}-\mathrm{H}$, whereas absorption at $788 \mathrm{~cm}^{-1}$ shows out-plane vibration of $=\mathrm{C}-\mathrm{H}$.

Sharp increase in temperature of fabric specimen was observed in first 3 min with the applied voltage which was then stabilized after reaching a plateau, as shown in Figure 5, and it shows the ease with which heat could be generated by employing a reasonable $\mathrm{DC}$ voltage.

Power density $P_{D}$ (volume specific power) is the amount of power (time rate of energy transfer) per unit volume. It is then also called volume power density which is expressed as $\left(\mathrm{W} / \mathrm{m}^{3}\right)$. Volume power density is sometimes an important 


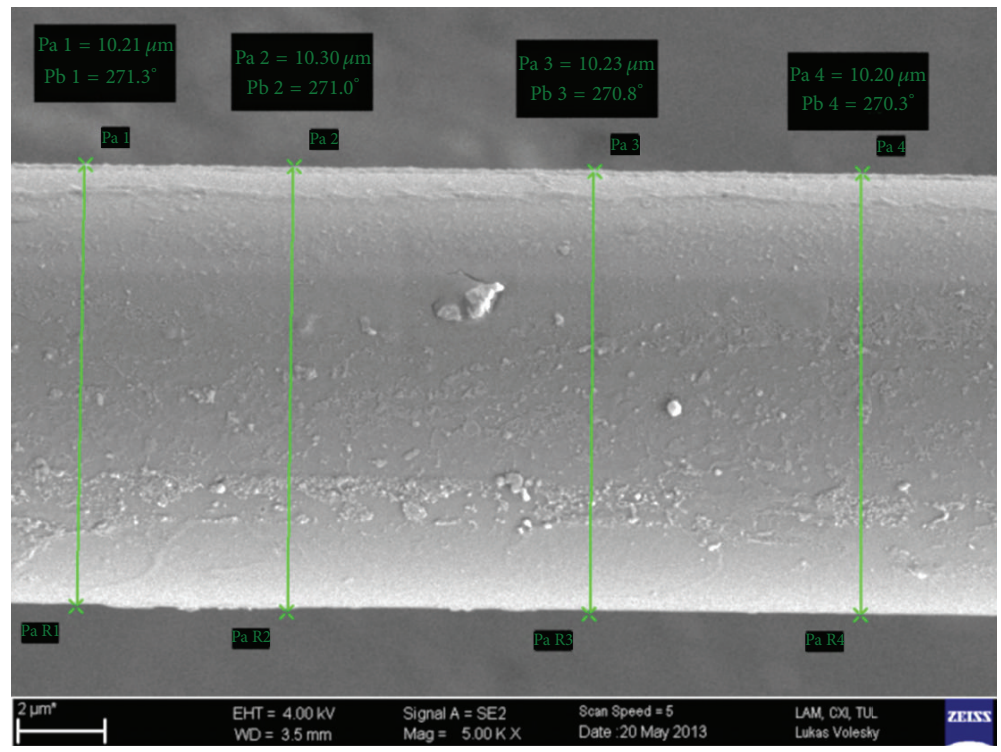

(a)

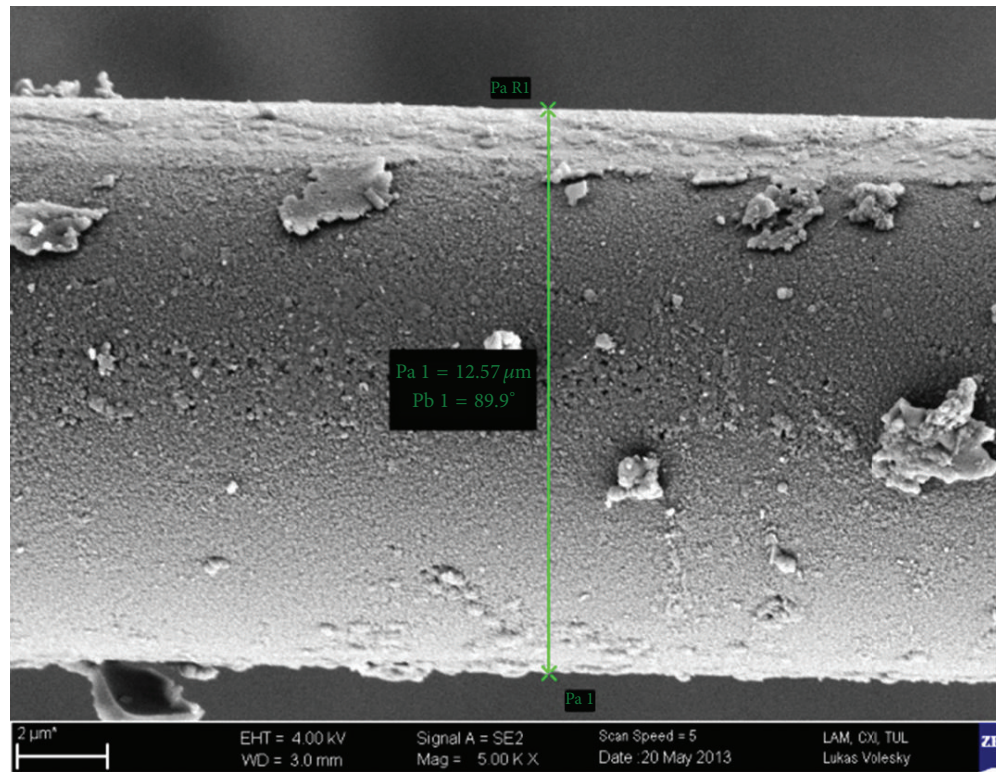

(b)

Figure 1: SEM micrographs: (a) E-glass control and (b) E-glass coated with PPy using $\mathrm{FeCl}_{3} 0.6 \mathrm{~mol} / \mathrm{L}$.

consideration where space is constrained. In this study, power density $P_{D}$ is extremely linear function of voltage supplied to the PPy coated glass fabric as illustrated in Figure 6 and expressed as

$$
\dot{e}_{\text {gen }}=\frac{\dot{E}_{\text {gen }}}{v_{\text {element }}}=P_{D}=82.58 \mathrm{~V}-245.83 .
$$

Here, $\dot{e}_{\text {gen }}$ shows the power generated per unit volume, $\dot{E}_{\text {gen }}$ the quantity of heat energy, and $v_{\text {element }}$ the volume of the heating element which in our case is fabric.

However, temperature rise in fabric sample at equilibrium (after $3 \mathrm{~min}$ ) follows exponential function of voltage with
$R^{2}=0.9717$, that can be written as

$$
T=T_{0} e^{0.0795 \mathrm{~V}} .
$$

Here, $T$ is the temperature in $\left({ }^{\circ} \mathrm{C}\right)$ measured at equilibrium at applied DC $V$, and $T_{0}$ is the temperature of fabric at ambient conditions, which was recorded as $22.3^{\circ} \mathrm{C}$ for this experiment.

\section{Conclusion}

For the specimen the rate of change of temperature has two distinct phases: an initial sharp rise during the first $100 \mathrm{~s}$ or so, followed by a leveling off to plateau at approximately 300 to $400 \mathrm{~s}$. During the first phase, the electrical energy input to 


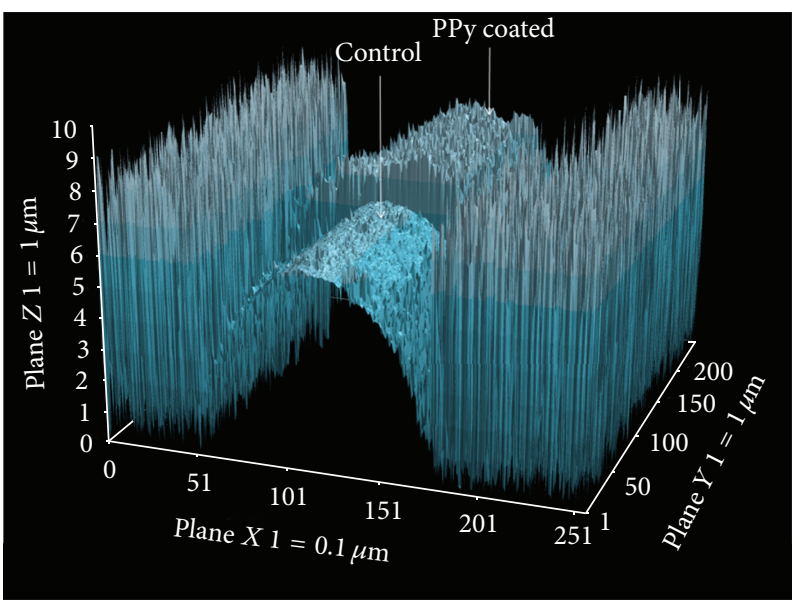

(a)

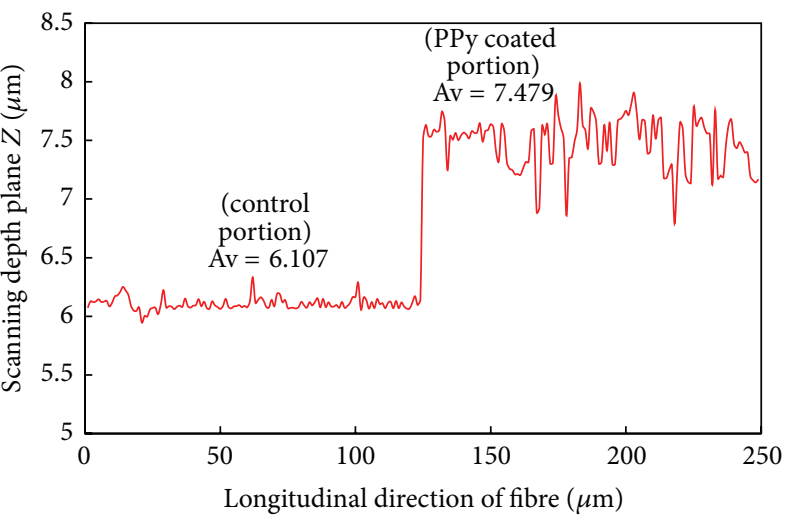

(b)

FIGURE 2: The surface morphology of the PPy coating using $\mathrm{FeCl}_{3} 0.6 \mathrm{~mol} / \mathrm{L}$ studied by Laser confocal microscope (a) describes the surface morphology and (b) comparison of thickness.

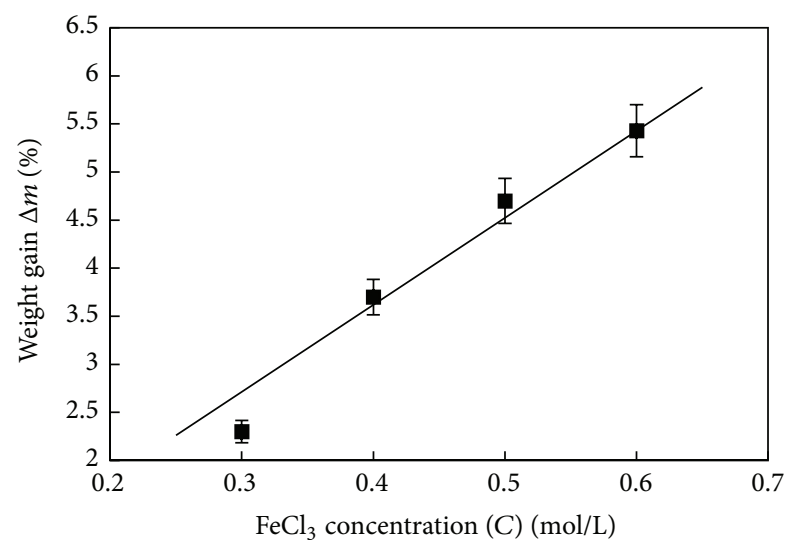

FIGURE 3: The dependence of weight gain by glass fabric after coating by PPy.

the samples exceeds the combined thermal energy losses in the forms of convection, radiation, and conduction and hence, the temperature rises. As the temperature rises the heat losses increase until they are balanced with the heat generated and the temperature of the fabric stops rising. This is the stable temperature of the fabric under the applied voltage.

The electrical power supplied to the specimen that ultimately converts into thermal power follows a linear dependence on the DC voltage supplied, whereas rise in temperature of the specimen is a power function of supplied voltage.

The estimated cost to produce PPy coated E-glass fabric at laboratory scale was roughly calculated as less than Euro $1.15 \mathrm{per} \mathrm{m}^{2}$, and this kind of heat generating industrial textile can find its practical application as heating systems for small and medium spaces as well as for the drying of wet cement in civil works.

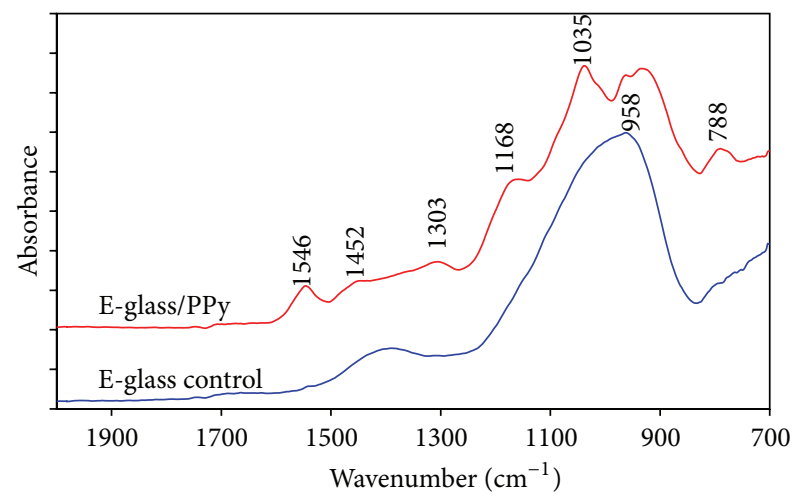

FIGURE 4: FTIR absorbance curves of E-glass control and E-glass coated with PPy.

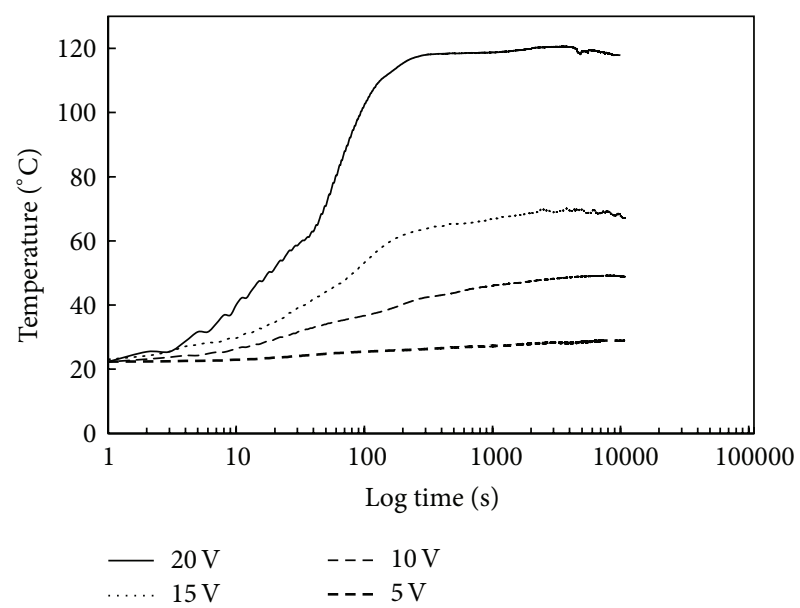

Figure 5: Escalation of temperature of E-glass fabric coated by PPy using $0.6 \mathrm{~mol} / \mathrm{L} \mathrm{FeCl}_{3}$ with increase in DC voltage $V$ and time. 


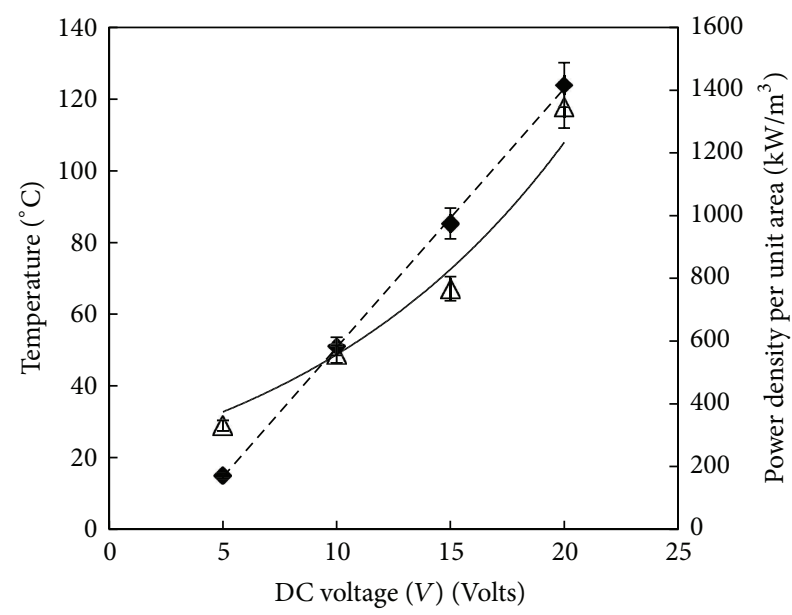

$\triangle$ Temperature

- Power density

FIGURE 6: Rise in power density and temperature of sample D4 as a function of DC voltage $V$.

\section{Acknowledgment}

This study was supported by student grant scheme (SGS) in Technical University of Liberec, Czech Republic.

\section{References}

[1] Y. Cao, P. Smith, and A. J. Heeger, "Counter-ion induced processibility of conducting polyaniline," Synthetic Metals, vol. 57, no. 1, pp. 3514-3519, 1993.

[2] C. K. Chiang, C. R. Fincher, Y. W. Park et al., "Electrical conductivity in doped polyacetylene," Physical Review Letters, vol. 39, no. 17, pp. 1098-1101, 1977.

[3] A. G. MacDiarmid, "Polyaniline and polypyrrole: where are we headed?” Synthetic Metals, vol. 84, no. 1-3, pp. 27-34, 1997.

[4] J. Edenbaum, Ed., Plastics Additives and Modifiers Handbook, Van Nostrand Reinhold, New York, NY, USA, 1992.

[5] T. Lin, L. Wang, X. Wang, and A. Kaynak, "Polymerising pyrrole on polyester textiles and controlling the conductivity through coating thickness," Thin Solid Films, vol. 479, no. 1-2, pp. 77-82, 2005. 

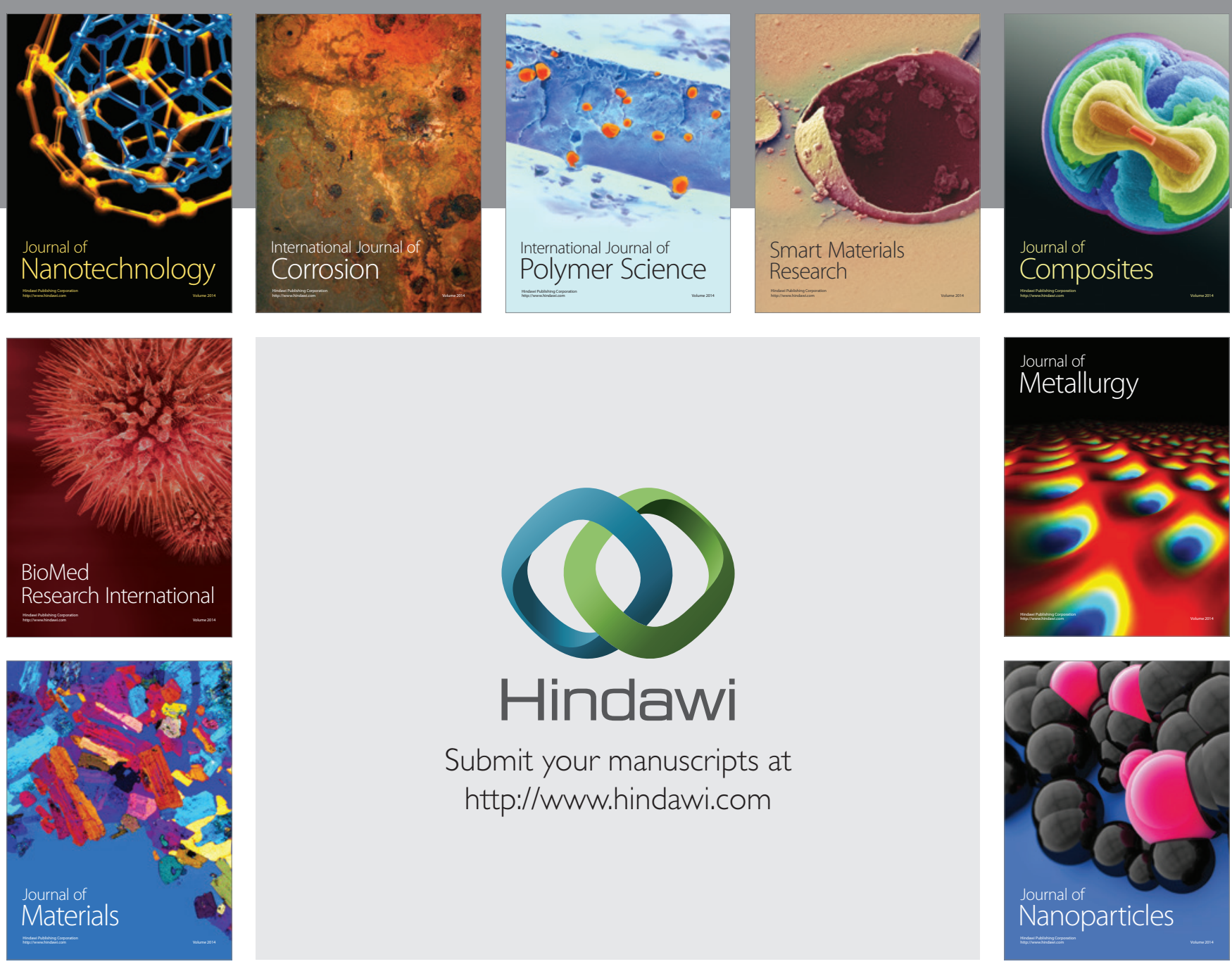

Submit your manuscripts at http://www.hindawi.com
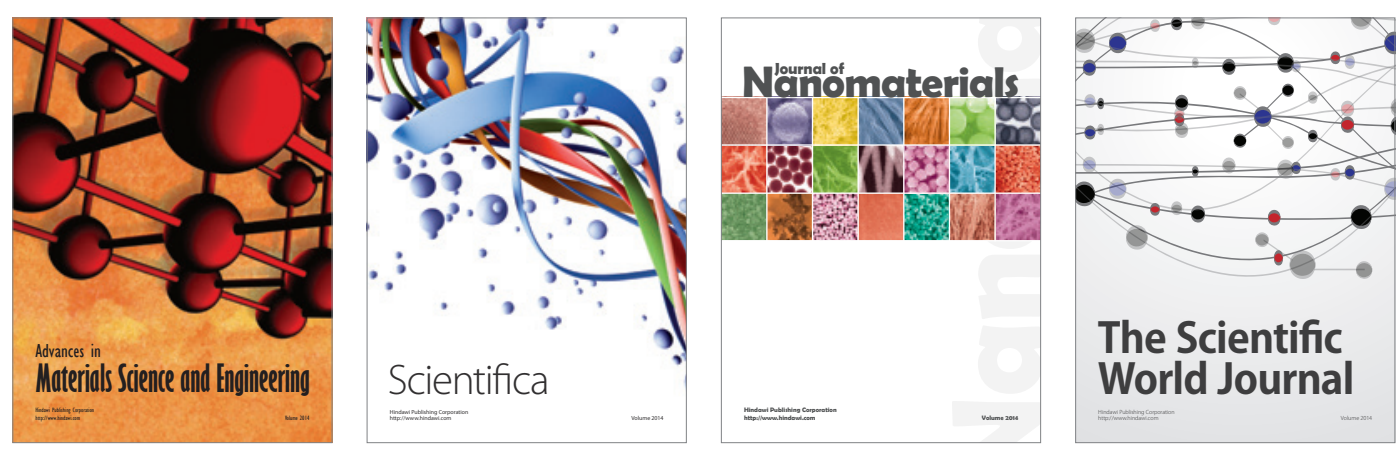

\section{The Scientific World Journal}
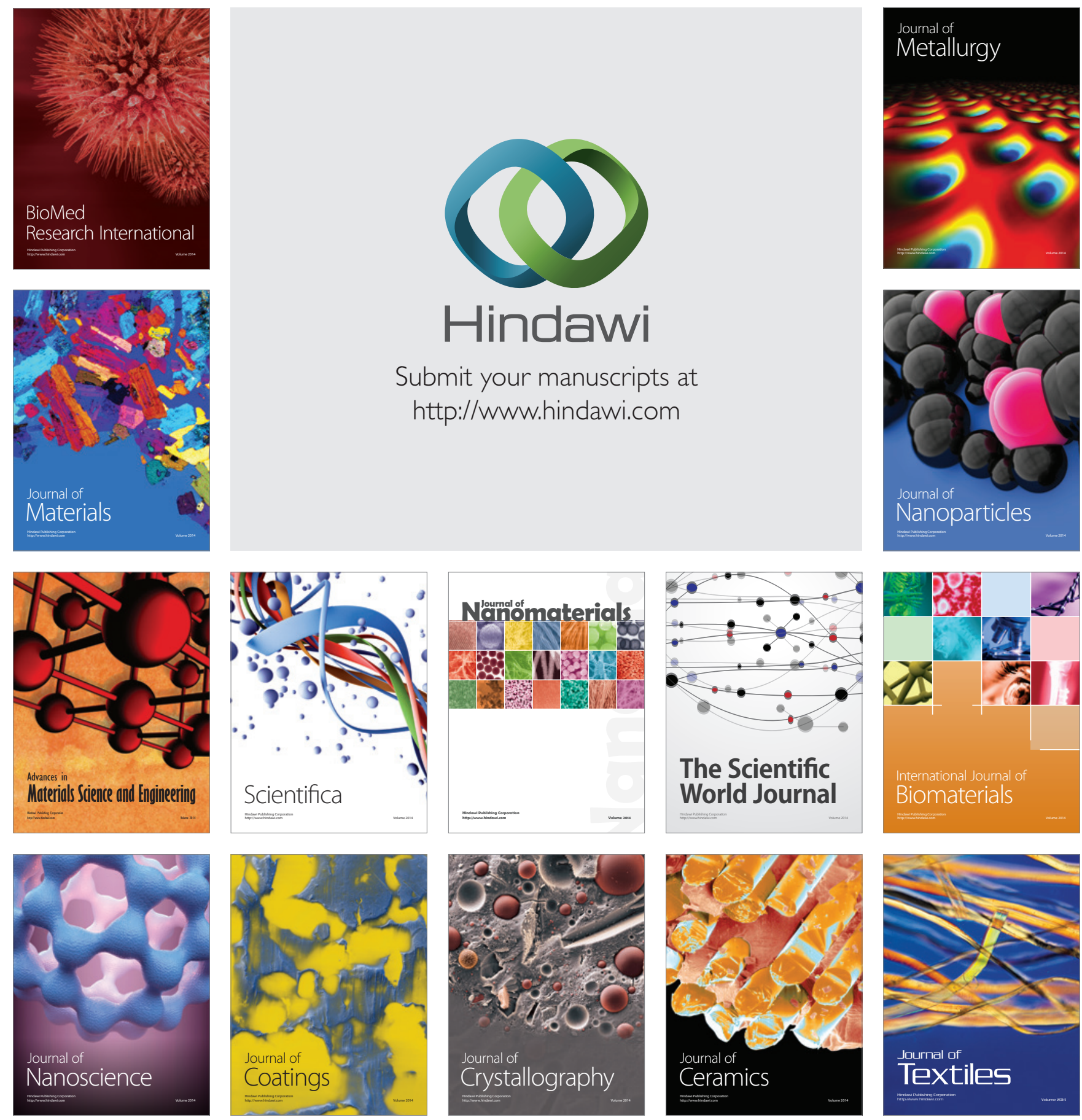